\title{
MAKING USE OF SOCIAL PROTOTYPES: FROM FUZZY CONCEPTS TO FIRM DECISIONS
}

\author{
Paula M. NIEDENTHAL and Nancy CANTOR \\ Institute for Social Research, University of Michigan, Ann Arbor, MI 48106, USA
}

Received August 1983

Revised November 1983

\begin{abstract}
Evidence suggestive of the 'fuzzy' structure of many natural language (as opposed to scientific) categories is reviewed. Examples are drawn from categorization schemes for objects, such as cars, fruit or tables, as well as for people, such as extroverts, hippies and fraternity types'. It is argued that despite the fuzziness of natural categories, there is orderliness and utility to these concepts, as well. Specifically, natural categories are organized around prototypes, representative category members, which serve as reference points for the category. The identification of category members involves a prototype-matching process. Prototypes about 'kinds of people' can be very useful in decisions about people with whom to affiliate and places in which to live. This process was illustrated by reference to a case analysis of students' preferences for university housing. It was concluded that natural categories may well be fuzzy, but they are far from being dispensible.
\end{abstract}

Keywords: Natural categories, Prototypes, Self-monitor, Distinctiveness, Interpersonal goals, Self-to-prototype matching heuristic.

\section{Natural categories for people and objects}

It would be tiresome to correct ourselves each time that we offhandedly referred to mushrooms and tomatoes as ingredients in a 'vegetable salad'. If we have invited our friend the botanist to dinner, however, we can scarcely avoid a confrontation on this issue. Predictably, the botanist hastens to inform us that the salad, given these ingredients, is a fruit-fungus-vegetable salad, and that if we chose to add watercress the name would sound even less appetizing. Meanwhile, the other dinner guests roll their eyes with impatience, thinking: "Are tomatoes sweet? No! Do I pour Italian salad dressing on bananas? No! For all practical purposes mushrooms and tomatoes are, quite obviously, vegetables ... I think."

The proverbial 'error', that of classifying tomatoes and mushrooms as vegetables, and the uncertainty experienced in the process of classification illustrate some differences in the genesis, structure and function of formal scientific categories on the one hand and natural categories, the means by which individuals informally organize their perceptual and conceptual environments, on the other. That is, botanists have invented a quite orderly and comprehensive taxonomy based on defining morphological and/or genetic attributes in order to organize 
their subject matter and to guide the field as a whole. But, day to day, non-experts often find themselves unable to follow the rules of tightly-structured taxonomies. Instead, they form 'fuzzy' natural categories calling, for example, 'things that go in salads' members of the vegetable category $[19,33]$. Natural categories blur the distinctions between classes of objects, breaking down the neat structure of those scientific taxonomic classifications. When non-experts classify fruits and vegetables, and other objects for which experts can find distinguishing defining features, those non-experts tend to partition the objects not on the basis of the select, criterial features, but rather on the basis of a larger array of characteristic features. As a result, the boundaries between categories are by no means clear - Is a tomato really more like a fruit (apple, pear) than a vegetable (spinach, peas)?

The fuzzy structure of natural categories, and the flexibility with which they are utilized by non-experts, becomes even more apparent in the study of classification systems based on attributes of people: for example, taxonomies of personality traits, mental illness, intelligence. In those domains, even the 'experts' have had considerable difficulty carving up the stimuli into well-defined taxonomies (see, for example, $[11,17]$ on psychiatric diagnostic taxonomies; [54]; [8] on the classification of personality traits; [37] on the concept of intelligence). This fuzziness persists in the classification of people even when one might assume the existence of some criterial features: Consider distinctions between men and women, masculinity and femininity $[5,35]$. If the scientists cannot always distinguish apples from oranges in the social environment, it is hardly surprising that the casual observer repeatedly perceives and employs imperfectly structured person, situation and object categories.

The importance of the conceptualization of natural categories, social categories in particular, as fuzzy sets is its prescription for studying how the social observer responds to the environment. Predictions about people's behavioral uniformity and variability are more sensitive if the cognitive 'rules' by which individuals assess social stimuli and regulate their actions accordingly are explored.

The theme of the present review, however, is that the fuzziness of natural categories does not imply their messiness or uselessness. In support of this point, we will draw on research literature concerned with the structure, mental representation and function of natural categories in a wide variety of domains involving animate and inanimate 'objects'. Our examples will range from categories for birds, fruit and cars to those used to sort extroverts, hippies and fraternity types. We will begin with a discussion of the 'fuzzy' but, nonetheless, orderly structure of natural categories and then turn to various models for the mental representation of knowledge about categories. Finally, in order to document our message about the utility of natural categories, we will present a case study analysis of an everyday decision-making task-choosing university housing-in which fuzzy natural categories are implicated in the observed choice preferences.

\section{The structure of natural categories}

Following Wittgenstein [55], linguists, philosophers and psychologists alike have recognized that natural concepts do not partition objects, animate or 
inanimate, into nicely-bounded categories (cf. [44]). This line of thought represents a departure from the traditional, classical view of categorization. The classical approach maintains that all members of a category share a single, complete set of defining features. Such a principle implies that the clearly-defined categories addressed by this view share at least four properties. First, category membership is based strictly on an all-or-none criterion; that is, possession of a set of singly necessary, jointly sufficient characteristics. Second, since all members share a set of common features, all members are equally suitable representatives of the concept. Third, categories are disjunctive; at one level of inclusiveness in a taxonomy, no single object can enjoy membership in more than one category simultaneously. Finally, since all defining features are necessary, each is equally salient in determining class membership.

The properties assumed to be true of categories and taxonomies, according to the classical view, are in fact characteristic of very few sets of stimuli in the environment that are naturally and quite consensually clustered together. It follows, then, that the classical view is altogether incompatible with the study of social concepts. As it happens, the state of the social world is not one of clearly partitionable stimuli; and so the imprecisions that are inherent in the environment get reproduced in the organization of objects into natural categories and taxonomies (see [15]).

\subsection{Fuzziness}

It is clear from the work of Rosch and her colleagues (see [43]) that people usually agree about the nesting of natural object categories into hierarchies. These taxonomic structures typically possess at least three levels of inclusiveness or abstraction. A representative object taxonomy may include categories which vary in abstractness, such as these: vehicle-automobile-sports car. Despite their relatively organized and consensual structure, however, natural taxonomies suffer from a property described as imperfect feature nesting. Frequently, an attribute considered highly associated with membership in a given category, such as the feature 'flies' with the concept 'bird', is not true of a member of a more specific sub-category, such as the category 'chicken' in this example [46].

The ill-defined quality of natural taxonomies - as seen in the natural category hierarchies - is also illustrated in the horizontal structure of natural categories; the manner in which objects are classified at any given level of abstraction [43]. Natural categories seem to be 'fuzzy sets'; category membership is not determined by the possession of a set of necessary and sufficient defining features. If a tomato is a fruit, why isn't it sweet as most fruits are? Is sweetness a defining feature of the category fruit? Probably not. We run in circles if we try to state absolutes about natural categories. Instead, stimuli seem to be grouped together to form a general category because they share many features that are perceived to be highly correlated with membership. So, just as tomatoes do not possess the quality 'sweet', all 'fruit' are not required to share the entire set of characteristics which are typical of that category [48]. Moreover, all characteristics are not equally common in the category. The property 'sweet' is more central to the concept fruit, for example, than is another attribute, such as round. It is the case, therefore, that 
any one category may possess both highly representative and unclear or borderline category members; members vary along a continuum of perceived goodness of fit or prototypicality in a category $[33,45]$.

Since natural categories are not defined by a set of necessary and sufficient features, the boundaries between perceptually or conceptually similar concepts are not always well-specified. Consider how often our inability to comprehensively cluster stimuli into orthogonal groups is reflected in the language we use in reference to dubious or borderline cases: objects are 'reddish'; literature is 'Kafkaesque'; foods are 'sweet-sour'. The implication is that closely related categories frequently overlap as the continuum of features that facilitate specification of membership in one category fades into that of another category. There is thus no absolute demarcation between fruits and vegetables; the features of one concept are shared extensively with the other.

The conceptualization of natural categories as fuzzy sets nested in loosely structured taxonomies is perhaps even more suitable when applied to the study of social categories. One predominate categorization scheme by which individuals naturally cluster 'kinds of people' is based on personality characteristics which are inferred in the process of observing and attributing causes to social behavior [26, 47]. Cantor and Mischel [15], for example, worked with four abstract person categories (Extroverts, Cultured People, People Committed to a Cause, and Emotionally Unstable People). These categories were chosen because they frequently emerge as basic factors in studies of personality traits [38]. Cantor and Mischel [15] used these abstract categories in order to generate four person taxonomies: they asked students to cluster each of 32 more specific person categories under one of these four abstract categories. For example, the superordinate category cultured persons was consistently used to incorporate the two less-inclusive categories, man-of-the-world sophisticate and patron of the arts. These middle-level categories were then further divided into more specific subcategories (e.g., man-of-the-world was divided into gourmet and world traveler). However, when asked to describe the features common to members of each category, subjects listed features as true of man-of-the-world, which were not necessarily seen as true of the gourmet, for example; so the imperfect feature nesting property is clearly characteristic of person hierarchies. Furthermore, from the point of view of a classical theorist there are two additional 'problems' with person taxonomies.

First of all, within a person taxonomy (e.g., People Committed to a Cause) any particular subcategory, such as fighter against child abuse, may seem to fit well within more than one higher order category (religious devotee or social activist). And secondly, a member of a given category may be an equally representative member of another, related category at the same level of abstraction. The fighter against child abuse may also be a save-the-whales demonstrator. Neither circumstance is tolerated by the classical view of categorization which asserts that concepts in a taxonomy should be disjointed, with no overlapping elements. In the person domain, however, it would not be difficult to think of an individual who is both a religious devotee and a social activist; both a patron of the arts and a man of the world sophisticate. 
One should not be fooled into thinking that these are just poorly constructed taxonomies, for the overlap between person categories within a given taxonomy is true even when one considers more carefully derived 'scientific' person taxonomies, rather than the 'everyday variety' used by Cantor and Mischel [15]. For example, Cantor et al. [17] worked with psychiatric diagnostic categories and observed the same imprecisions: imperfect feature nesting, non-rigid category boundaries, characteristic rather than criterial category features, and a continuum of category membership from clear to 'borderline' cases.

Since person categories are fuzzy and overlapping, category membership is a matter of degree, but fortunately the social environment is replete with helpful indicators. Consider the process of identifying an extroverted person. Most extroverts, people tend to agree, are friendly and gregarious. Still, some are more accurately described as aggressive and energetic, and not particularly warm or sociable. As a concept, extrovert contains both 'classic' as well as atypical, dubious examples. Moreover, complicating matters further is the information made available to the perceiver by the ongoing behavior of a target individual. Since behavior, which implies the existence of particular personality characteristics, varies as a function of context, class membership depends in part upon the frequency, or proportion of extroverted behaviors across time and in different social situations. We are all aware that extroverts can act in an introverted fashion within a formal, stressful or unfamiliar context. In fact, a display of extroverted conduct (talking, laughing and socializing) in the context of a funeral, for example, may be inappropriate enough to warrant a shift in the classification of an individual from "extrovert" to 'emotionally unstable person'. Ascertaining category membership for person categories requires a probabilistic assessment; one that may shift with increasing exposure to the target individual.

\subsection{Orderliness}

At this point, the reader may wonder how psychologists can consider natural categories to be orderly and useful. There do not seem to be criterial features for natural categories; similar categories overlap considerably in characteristic features and, thus, in membership. But, we would argue, if natural classification systems are not flawless in their structure, neither are they haphazard or messy.

Theories of the cognitive representation of natural categories provide a framework within which to understand the orderliness of natural categories as they are used by the average perceiver of the physical and social environment. The study of categorical representation has been fruitfully guided by the assumption that it is the most prototypical or central element of a concept that is held in long-term memory [43]. So, even while natural categories are not precisely circumscribed and have tremendous heterogeneity of membership, they can be simplified by "thinking of prototypes." Prototypes are defined not as the most frequent per se but the most representative members of a class; the elements that best symbolize the essential meaning of the category. The significance of prototypicality has been demonstrated in studies of perceptual learning [39, 41], inductive reasoning [27], and semantic memory [49]. Moreover, support for the argument 
that natural categories are stored in memory as prototypes derives from the literature on the organization, speed and accuracy of classifying objects that vary along a continuum of representativeness as members of a certain category [49]. In addressing the first issue, Mervis, Catlin and Rosch [34] found that highly representative members of a category are most likely to come to mind first when subjects are asked to list as many instances of a concept as possible within a time constraint. For example, when required to generate members of the category furniture, individuals will list chair and sofa long before they list hatrack and reading lamp. In addition, subjects are quicker to classify a prototypical target example of a category than an atypical example $[42,43]$. That is, robin and sparrow are identified as members of the category bird more rapidly than are eagle or hawk, which are, in turn, classified more rapidly than are goose or chicken. Finally, 'errors' or disagreements in classification per se also decrease as representativeness of the target example increases.

As the central tendency or focal point of a concept, a prototype can serve as a reference point; a standard of comparison to which stimuli can be compared to facilitate judgment of goodness of fit in a plausible category. The implication here is that while there are no easy and diagnostic rules to insure proper categorization for fuzzy categories, target instances can be classified on the basis of similarity to a prototype [53]. For instance, an object may be judged as a typical or representative class member because it possesses many characteristics considered to be highly correlated with category membership [45]. So, an apple is perceived as a good representative of the category, fruit, because it shares many of the characteristics with one's prototype of fruit (e.g., is sweet, round, juicy, has seeds, can be peeled, grows individually, makes good pies, and so on). To the extent that an object deviates from the prototype it is considered atypical, though still a member of the category. Some grapes don't have seeds, they grow in bunches and the peel is imperceptible; thus, grapes are not particularly good representatives of the concept, fruit. Tomatoes, however, are even poorer examples, since they are not sweet, and are found more often in the company of vegetables than in pies. In other words, on the basis of similarity-matching to prototypes, target instances can be reliably categorized, even in fuzzy categories [48]. This rule makes the categorization process more orderly and consensual, and helps us to address the heterogeneity of category members-instances are judged to be more or less prototypical based on possession of features which, themselves, are only correlated with membership in the category.

In the social domain, types of people can also be reliably judged to vary along a continuum of goodness of fit in a category, by comparing a single instance to the standard or prototype (see [9] for prototypicality judgments about extroverts and intelligent persons; [20] for a discussion of occupational prototypes; [6] for prototypes of the 'elderly'; [29] on presidential candidate prototypes). If one imagines a series of concentric circles representing goodness of fit, or distance to the central prototypes, then, in our extrovert example the aggressive, energetic extrovert would lie in the periphery while the friendly, gregarious extrovert would be more centrally located. Given a standard or prototype represented in memory, person and object categories no longer seem so messy; despite the variability of 
members within categories, individuals can not only identify but also evaluate the representativeness of a particular example of a concept.

Similarity-matching to prototypes relieves some of the problems of classification caused by the absence of criterial features in fuzzy natural categories. But, identifying what a target instance is requires that we also ascertain what it is not. This process of differentiation is made difficult in natural classification systems by the overlap of categories within a single taxonomy. However, once again probabilistic rules of judgment can be reliable and orderly, though not infallible. For example, Rosch \& Mervis [45] found that subjects used contrast sets of similar, overlapping categories in the prototype-matching process-a tomato is an equivocal, borderline exemplar of the category fruit, not only because it has few of the prototypical features of fruit (it isn't sweet, cannot be peeled, isn't found in pies), but also because it shares as many features common to the contrast category of vegetable (it goes in salad, is tart, can be stewed).

Similarly, in the person domain, Cantor [9] found that subjects used the contrast category introvert quite effectively in distinguishing 'good' from 'poor' examples of the category extrovert. The aggressive extrovert, for example, may be a peripheral member of the category, extrovert, because 'coldness' is more typical of introverts than of extroverts. Even though extroverts and introverts are actually quite overlapping person categories, prototype-matching to prototypes for both categories can help an individual make a probabilistic estimate of 'best fit' for a target instance who seems to share features characteristic of both categories. Another trick that individuals use to assess category fit in fuzzy person categories, is to consider the context in which an individual exhibits category-relevant behavior [9]. Back in the context of a funeral, our extrovert may be quite easily differentiated from an emotionally-unstable person precisely because he exhibits an introvert's restraint - the use of situational cues can be very instrumental in resolving conflicts in person categorization.

Person categorization is especially difficult not only because the categories within a single taxonomy overlap so much, but because it is quite reasonable to expect an individual to 'belong' to many of these interrelated categories at once. With regard to this aspect of the fuzziness of person taxonomies, Cantor [9] found that individuals seemed to relieve some of the ambiguity in categorization (when forced to make a single categorization for a target) by assessing the relative salience of the category-relevant features in the target person's total personality. A 'good' extrovert, then, not only has many typical extroverted features (and few introverted qualities), but these extroverted features are also quite robust, being manifest in many situations, over time, and in a variety of behavioral forms.

In order to use contrast sets effectively in the similarity matching process, it is useful to know that categories close together in a taxonomy (i.e., in one branch of a multiple-branching taxonomy) are the ones likely to share similar prototypes and, thus, overlap in category membership. The absence of perfect feature nesting in natural taxonomies is a bit worrisome in that regard. Perfect feature nesting would certainly ensure the appropriateness of choosing contrast sets from a single branch of a taxonomy. In the context of imperfect feature nesting, such an algorithm for choice of contrast set could be a mistake. Cantor et al. [17] 
observed such a problem in their study of psychiatric diagnosis - patients diagnosed as manic-depressive-manics actually overlapped in clinical features most closely with a diagnostic category from another branch of the DSM-II taxonomy (paranoid schizophrenic). However, on the whole, that pattern of inter-category overlap is not observed. Instead, typically, the closer any two categories are to each other in a taxonomy (either vertically or horizontally close), the more their prototypes will tend to overlap in characteristic features (e.g., [16]). As a consequence, it is usually reasonable to assess degree of category fit by comparing an instance to the prototypes for multiple categories, all chosen from within a single region of a taxonomy. Again, natural categories and taxonomies are fuzzy, not messy; likewise, the rules of categorization are probabilistic, not unreliable.

\section{The representation of natural categories}

By now it should be clear that despite the fuzzy structure of many natural categories, prototypes seem to be highly memorable reference points or standards of comparison against which 'objects' in the environment can be compared in order to facilitate classification [43]. And, if prototypes provide order in natural categorization, then it is reasonable to assume that natural categories are represented in long-term memory by prototypes. Consequently, the form that prototypes may take in long-term memory is a point of great interest [48].

According to one approach, the prototype is represented as one or more 'classic' examples of old or known concept members [48]. An object is perceived as a good instance of a particular category if it represents a close match to at least one prototypical exemplar. To illustrate, Bob might promptly label a casual acquaintance, Mary, an 'introvert' because she displays behaviors and characteristics that are consonant with those of an old friend, Frank, who represents a classic introvert. In this case, Frank possesses most features that Bob believes are highly associated with the introverted personality. Moreover, Frank is a concrete, highly imageable standard of comparison. Since Mary is perceived to act like Frank in his essential, introverted ways, Mary is classified as an introvert - an introvert 'just like Frank'. Bob, it should be noted, may also be familiar with two other 'classic' introverts, Rudy and Paul, who symbolize different introverted 'types'. For example, Rudy might be an anti-social, misanthropic and cold introvert, while Paul is a submissive, dependent and unaggressive introvert. In this case, all three friends represent "typical introverted types" for Bob. To the extent that people encountered in his world match one of the three standards, Bob would classify them as introverts. But, what about atypical cases-how does the exemplarprototype help in classifying them? Following this approach, the atypical case would provide a poor match to any one of the category exemplars - so, Joe, Bob's 'introvert' friend who is prone to displays of sudden extroversion, wouldn't match very well to Frank, Rudy, or Paul. And, Joe's occasional extroversion might lead Bob to retrieve from memory some exemplars of the extrovert category, as well. The atypicality here would be symbolized by the poor and conflicting matches to exemplars from the two contrast categories. The decisive criterion for labelling 
Joe as 'basically' an introvert (with some extroverted tendencies) would center on order of retrieval of category exemplars - Joe's features call to mind introvert exemplars before extrovert exemplars [48].

The exemplar-prototype representation preserves separately in memory a variety of concrete instances of the category; the representation nicely illustrates the heterogeneity of category membership. Another well-researched prototype representation, the summary-prototype, captures the potential for heterogeneity of members without directly storing in memory the different instances themselves $[14,46]$. This view holds that a concept is represented as an abstract summary representation or theoretical ideal that consolidates all of those characteristics believed to be correlated with membership in the category. The most representative members of a category, then, share many common features with the concept prototype and few overlapping characteristics with prototypes from other contrasting categories. Of course, in reality, the entire set of attributes included in the prototype representation is found in few, if any members of the category; and even representative category members may possess different subsets of the features in the summary prototype (thus forming a family resemblance structure [55]). The features in the summary prototype also vary in how representative of category members they are; that is, some features are common to most members (e.g., has feathers, has wings for the category, birds), while other features are typical of only a few category members (e.g., nests in trees, migrates in winter). In this sense summary prototypes accommodate to the fuzziness of natural categories by allowing for a continuum of 'representativeness' or prototypicality of member instances.

The summary prototype representation has been quite well-researched in a number of domains; prototypes have been generated for categories ranging in content from tables and cars [46] to religious devotees and comic-jokers [15] to librarians and waitresses [20] to senior citizens, elder statesmen and 'grandmotherly types' [6] to parties and religious ceremonies [16]. Typically, the prototype for a category is generated by asking a group of individuals to list the features common to and characteristic of category members. Each feature generated for a category, then, has a frequency score representing the number of individuals in the group who thought of that feature for that category-the frequency numbers are taken as indications of the degree of association of the feature with the category members. A consensual summary prototype can be formed by eliminating from the feature list for the category, idiosyncratic features that were listed by very few individuals (e.g., features listed by less than 10 or $20 \%$ of the group). Of course, individuals differ in their prototypes for particular categories; there is usually good, but not by any means total agreement in these feature lists. There are few, if any, criterial features which would come to mind immediately to all participants when describing most natural categories.

To illustrate, consider the prototype of the concept, tree. People spontaneously describe trees as having green leaves, brownish bark, a trunk, and ascending branches. After further consideration they will mention seasonal coloring and loss of leaves, or fruit in various forms. Some people may also refer to the "fact" that trees provide shade, can be climbed and offer shelter for animals and birds. And 
occasionally included in the ideal abstract summary is an indication of the standard size and shape (circular-shaped crown on a single trunk) of trees. Apparently, immediately available in memory are those features of the prototype (e.g., green leaves, brownish bark and trunk) that are most fundamental to the concept of tree, or true of most members of the category. The characteristics 'display seasonal coloring and loss of leaves' or 'can be climbed' are less central, and less often cited as essential features. Since to the non-botanist trees do not all share a small set of criterial features, even typical trees may not possess all of the characteristics that are part of the ideal summary. Note that trees that do closely match the category prototype, such as elms, oaks and maples, also share many features in common with each other and do not overlap extensively with related categories (e.g., shrubs). On the other hand, cyprus, willows and palm trees are considered less representative; they possess fewer prototypic features and share much in common with other categories (plants).

The exemplar-prototype and the summary-prototype both seem to provide orderly and useful representations of the fuzziness of many natural categories. [In fact, as Armstrong, Gleitman and Gleitman [2] have argued, even well-defined natural categories have a continuum of prototypicality of membership based on possession of characteristic features in addition to criterial ones. Consequently, these prototype representations would be useful even in the identification of members of well-defined categories.] Which representation, then, is superior? The representation should provide a vivid, rich, and yet flexible portrait of category members, while simultaneously differentiating the category from other neighboring categories. Such a representation would be ideally suited for use as a standard of comparison in the identification of category members.

In considering the utility of each prototype representation, it is important to recall that people categorize objects and other people at many levels of abstraction or inclusiveness, depending upon their goals and purposes at the time. Perhaps the summary prototype and the exemplar prototype are useful as representations of categories at different levels of inclusiveness in taxonomies [12]. The work of Rosch and her colleagues on 'basic level' categories provides some support for this speculation. Rosch et al. [46], working with three level taxonomies, found especially vivid (imageable), rich and distinctive (nonoverlapping) summary prototypes for categories of common objects described at a middle level of inclusiveness. The summary prototypes for car and boat, for instance, were rich and distinctive, one from the other. Moving to a more concrete, subordinate level of categorization, the summary prototypes for sports car and station wagon were still quite rich and vivid, but overlapped considerably in characteristic features. Cantor and Mischel [15] found a similar pattern in their investigation of summary prototypes in person taxonomies, as did Brewer et al. [6] in their study of categories for elderly persons. These results suggest that the summary prototype representation is perhaps more useful (in the easy identification of category instances) for categories at a middle (or 'basic'), rather than subordinate level of inclusiveness. First of all, retrieving exemplars for categories at the more inclusive middle level might become a cumbersome procedure, since the categories are relatively broad. And, in any event, the summary prototypes of 
middle level categories are reasonably vivid and distinct. Exemplar-prototypes would seem to be perfect representations at the subordinate level, however. They are as concrete and vivid as possible and they emphasize the heterogeneity of categories. The exemplar-prototype would appear to be better-suited than the summary prototype to 'pull apart' overlapping categories at the subordinate level in these taxonomies.

Suppose our speculations prove correct, and people show a tendency to retrieve exemplars when categorizing at more specific levels, while relying upon the summary prototypes at slightly more inclusive levels of categorization. What does this suggest with respect to frequency of use of exemplars and summary prototypes, i.e., frequency of use of categories at subordinate versus middle levels of inclusiveness? This remains an open and complex question, of course. However, it does seem likely that the answer will depend upon the expertise of the categorizer in that domain, the amount of information available about the target instance, and the goals and purposes of categorizing at all in that context [7, 43]. For example, imagine a man enters a cheese shop and requests a pound of cheddar cheese. An obsequious salesperson, so inclined, might complicate matters by pressing the customer to pinpoint which of many cheddar varieties he desires. Is it Vermont, Wisconsin, goat's, or longhorn cheddar? Does he want it mild, medium, or aged? Suppose now the customer merely shrugs 'just plain cheddar'. The shrug might imply that the man has not accumulated relevant knowledge or experiences - the store of easily retrievable, distinctive exemplars - that would aid in further differentiating the category, cheddar. Alternatively, as Rosch et al. [46] note: "It is to the organism's advantage not to differentiate one stimulus from others when that differentiation is irrelevant to the purposes at hand" (p. 384). In other words, our immediate purposes often direct how we draw upon our knowledge and utilize existing categories. So, if our cheese-shopper were planning to bake a casserole imbued with spices pungent enough to overwhelm the flavor of cheese anyway, the difference between goat's cheddar and Vermont cheddar would certainly be insignificant. His shrug in such a case would imply indifference and not necessarily ignorance. Moreover, if the same cheese-buyer had entered a less specialized cheese shop, one in which all cheddars were considered alike and only one kind of cheddar was available that day, then categorizing at the subordinate level would be pointless at best.

Translating to the social domain, the specific subordinate level (represented by collections of exemplars) may be more useful for careful and detailed analyses and predictions about a specific person's behavior in a given situation. Higher-order, more inclusive categories (represented in memory by summary prototypes), on the other hand, may serve better to organize an overall impression of a group of people that can then be distinguished from other general classes $[15,24]$.

\section{The utility of representing natural categories by prototypes}

As suggested earlier, prototypes provide us with relatively simple and useful cognitive schemes that include much of what we need to know about representative members of the spectrum of natural categories. Person prototypes function in 
much the same way as object prototypes, offering the same route to swift and efficient processing of information in the social world [30, 31]. The kinds of predictions, judgments and inferences that individuals formulate about members of person categories, in contrast to object categories, however, may have more profound effects on the perceiver's behavior.

When it comes to 'things', our principal question is "What is it?" Take as an object of concern, a vehicle. If the vehicle presents itself with two tires, handlebars, a saddle, gears and handbrakes, while a motor, windshield and steering wheel are conspicuously lacking, the object probably jibes well with our prototype of a bicycle. In terms of behavior one could conceivably choose to ride or, given its state of repair, fix the bicycle. And, barring a bizarre past experience, this encounter would be relatively nonaffect-laden.

Now consider an interaction with an unfamiliar woman who has stopped us on the street to obtain directions to such-and-such a street which is, by chance, the street we are bound for. The woman brings with her a barrage of informative physical attributes - long straight hair, sandals, no make-up, a peasant blouse, unshaven legs, and a button that reflects an acute dissatisfaction with the current political administration. More abstractly, she seems uninhibited, friendly and relaxed. And she uses a peculiar style of speech flavored with an identifiable jargon. Chances are we will not bother to ask simply, "What is it?" Our evaluation may be as complex as the person we are interacting with. So, this time we might consider "What kind of person is she?" "Where is she going?" "Do I like her enough to accompany her, since I'm headed in the same direction?" Interestingly, such questions can be answered quite readily after only a brief encounter, and often with illusory feelings of certainty. Asked to form an impression of the woman at this point, we might conclude "She is a hippy-type who is probably going to such-and-such a street because there are many food coops on that street. Chances are she is in town to attend the anti-nuclear rally. Her political attitudes seem to correspond to ours, and besides, she is probably a pacifist, so walking her toward her destination would be safe enough and maybe enjoyable."

Given time, we might persist in our inference-making and, having categorized this woman as a hippy, predict her preferences in entertainment, music and friends. The use of person prototypes serves to create an almost instantaneous feeling of 'knowing' a mere acquaintance; categories help us to form impressions and plans of action and they limit the range of behaviors we can expect from a given type of person in a specific situation.

This excerpt from an article in the New York Times Magazine illustrates the kinds of categories and prototypes which people employ everyday for the purposes of identification, prediction and decision-making:

... You can suit your image in the same disgusting way you can do anything when money is no object. I have a friend in the automobile business whose name is Fred. He did it by going on a diet and then buying a white Jaguar for days and a blue Ferrari for nights. He had to go on a diet because a Ferrari is a thin car. When you drive a Ferrari, you don't 
have room for an extra pack of king-sized cigarettes, let alone your very own spare tire.

"The Jag is an open car, that's why it's for days", Fred says. "The Ferrari is a coupe and that's why it's for nights. But you talk about this image thing - you can pretty much typecast who will own what kind of car. If you're a pilot, for instance, you buy a Saab. Guys who are into mechanical weirdness always buy Saabs...."

(New York Times, Sunday, July 10, 1983).

Sometimes, of course, such inferences, based on superficial appearances or brief contact, are inaccurate and even hurtful. Research on stereotyping has demonstrated the pernicious effects of overgeneralizing from exemplars and prototypes in a way that homogenizes perceptions of all target instances [22]. Individuals all too easily relieve the burden of discriminating atypical from typical category members by "filling in missing features" with prototypic ones - "all Germans are industrious, dogmatic, and nationalistic, aren't they?" $[18,22]$. While prototypes may be very useful in this regard, the use of prototypes, ironically, may encourage perceivers to overlook the actual heterogeneity within natural categories, as well as the overlap between categories [40]. So, we say "children will be children" or "those two are as different as night and day". Prototypes do help organize social stimuli and smooth over the complexities of the social world, though perhaps a bit too well.

Despite these hazards involved in the over-use of person prototypes, we feel that these constructs can be of substantial value in everyday life. Person prototypes do facilitate social interaction by helping us form impressions of other people quickly and then to plan our interpersonal behavior, accordingly. The impressions are sometimes inaccurate, as in the case of fallacious stereotyping. Yet, particularly when one has been careless, it is still possible to acknowledge one's errors during the course of a social interaction and correct them (i.e., substitute another, more appropriate prototype). In order to initiate behavior people need to reduce the cognitive strain from the abundance of incoming social stimuli; person prototypes are quite useful generalizations in that respect.

Such prototypes might also serve another useful function in naturalistic settings. That is, prototypes supply the information and focus we need to entertain everyday decision-making tasks. Suppose, for example, that you were agonizing over the attractive option of buying a Saab or a Ferrari. Now, having just 'learned' about the prototype for 'Saab-people' and 'Ferrari-people', you would be in a position to compare yourself to each person prototype and make a choice of the car which best fits your personality. Of course, our choices of products, vacations, houses, friends, and so on are determined by many factors other than personality suitability. Still, personality suitability is one pertinent factor in many decisions, and prototype-matching is, thus, an extremely functional heuristic. For that reason, among others, we suggest that person prototypes are knowledge structures of high utility in everyday life. In order to make this point more convincingly, we will turn now to a case study of the usefulness of person prototypes in a naturalistic decision task. 


\section{Case study}

In the present study, we [36] undertook an analysis of a social decision-making task in which we believed the utility of person prototypes could be powerfully demonstrated. The decision concerned selection of housing on the part of one-hundred and sixteen college freshmen at the University of Wisconsin. The University environment offers students a variety of living possibilities; seven major housing categories were identified: coed dormitory, single sex dormitory, fraternity and sorority, cooperative, apartment, rented house (flat with other students), or a rented room in a private residence. We were able to interview the students about factors underlying their decisions as they were in the process of deliberating between these options in the spring of their freshman year.

Our analysis of the role of person prototypes in decision-making (about housing) stemmed from the following observation: Person prototypes sometimes contain information about the settings that different kinds of individuals tend to occupy or enter [10]. For example, expectations about interactions with waitresses, professors or dentists derive in part from knowledge about the typical events, roles and behavioral demands characteristic of the (prototypic) situations in which these people are encountered $[1,20]$. Individuals often feel confident to predict who will be comfortable and successful in many contexts $[13,15,52]$. In so doing, they make use of prototypes of suitable person-in-situation combinations $[4,10]$.

On the basis of prior work on person-in-situation prototypes (e.g., [16], we reasoned that college students would share quite consensual impressions of the person-best-suited for the different housing situations. Moreover, we assumed that some students would be able to make use of those prototypes in order to rank order the desirability of living in each housing option, i.e., preferring the situation whose prototype best fit their own self-images [25, 32]. Our primary aim was to determine whether students used their knowledge of person prototypes to decide where they themselves would be most happy and comfortable and, if so, who could best make use of a self-to-prototype matching heuristic in this decision task. We attempted to outline a "prototype' of the individual who possesses and can utilize social knowledge in order to discriminate 'good' from 'poor' self-insituation matches.

Our first task was to see if these students actually had person-in-situation prototypes in this domain. For this purpose, we analyzed the richness and distinctiveness of a consensual person-in-housing prototype for each of the seven housing options. Next, we compared the individual's own housing prototypes to their self-concept descriptions, in order to assess the utility of a self-to-prototype matching rule in housing selection. Students were asked, as part of the interview, to rank order the seven housing options in terms of their preferences for university housing. The correspondence between an individual's self-to-prototype match and their preference rank order for each housing option provided the test of the matching rule. Ultimately, we looked at subsamples of students for whom a self-to-prototype matching rule was especially predictive of their housing preferences. 


\subsection{Consensual person-in-housing prototypes}

Consensual person-in-housing prototypes were of interest for two reasons: First, it was important for us to document that these students did, in fact, share rich, distinctive, and intuitively reasonable prototypes in this domain. Also, we wished to demonstrate that even these consensual prototypes, relatively global indicators of knowledge about housing, would have some bearing on the students' housing preferences.

The method employed to tap consensual person-in-housing prototypes was based on literature showing that individuals frequently describe the person who is best-suited for a situation in terms of characteristic personality traits [16]. To elicit the prototypes we gave all subjects a list of 100 trait adjectives covering a wide spectrum of personality domains [28]. They were asked to consider the housing situations separately, and for each one, to rate how accurately each adjective described the 'typical person who would be happy and comfortable living in this housing situation'. Subjects characterized their own prototype of the best-suited person by rating each of the 100 adjectives on a scale from one (not at all true) to eight (very true). ${ }^{1}$ Consensual prototypes were constructed by selecting the traits that were described as very true (ratings of 7 or 8 ) and those traits that were considered not at all true (ratings of 1 or 2) by at least $50 \%$ of the 116 subjects. Lists of the specific adjectives descriptive (i.e., definitely true and definitely not true) of each prototype are presented in Table 1.

Two aspects of these consensual prototypes are noteworthy here. First, the prototypes were indeed rich (on average, 14.7 traits rated as very true and 7.6 traits rated as not at all true for each prototype). However, the richness was not reflective of a bias to describe each prototype with the same traits; the prototypes were also distinctive. Table 2 presents the percentage of each prototype's descriptive traits shared by each of the other six prototypes. These figures clearly indicate that each prototype had both shared and unique features within the set of options. The prototypes also overlapped predictably and to different degrees: For example, the prototype of the person most comfortable in a single sex dormitory shared $69 \%$ of its descriptive features in common with the person best-suited for a coed dormitory, while the fraternity type overlapped in only $4 \%$ of its traits with the prototype for room in a private residence. This pattern of prototype richness and inter-prototype distinctiveness supported our assertion that prototypes can be useful in decision processes.

Encouraged by the integrity of these consensual prototypes, we first looked at the correspondence between the most frequently preferred housing options and the consensual prototypes which provided the best matches to the students' self descriptions. (Each student had rated him/herself on the same 100 traits used to construct the consensual prototypes.) For each housing option, we obtained a measure of average overlap between the students' self-concepts and the attributes

\footnotetext{
${ }^{1}$ We chose a structured, rather than a free-description procedure for prototype construction, so that the same 100 adjectives could be used in the self-description task. In this way, self-to-prototype match measures could be constructed easily.
} 


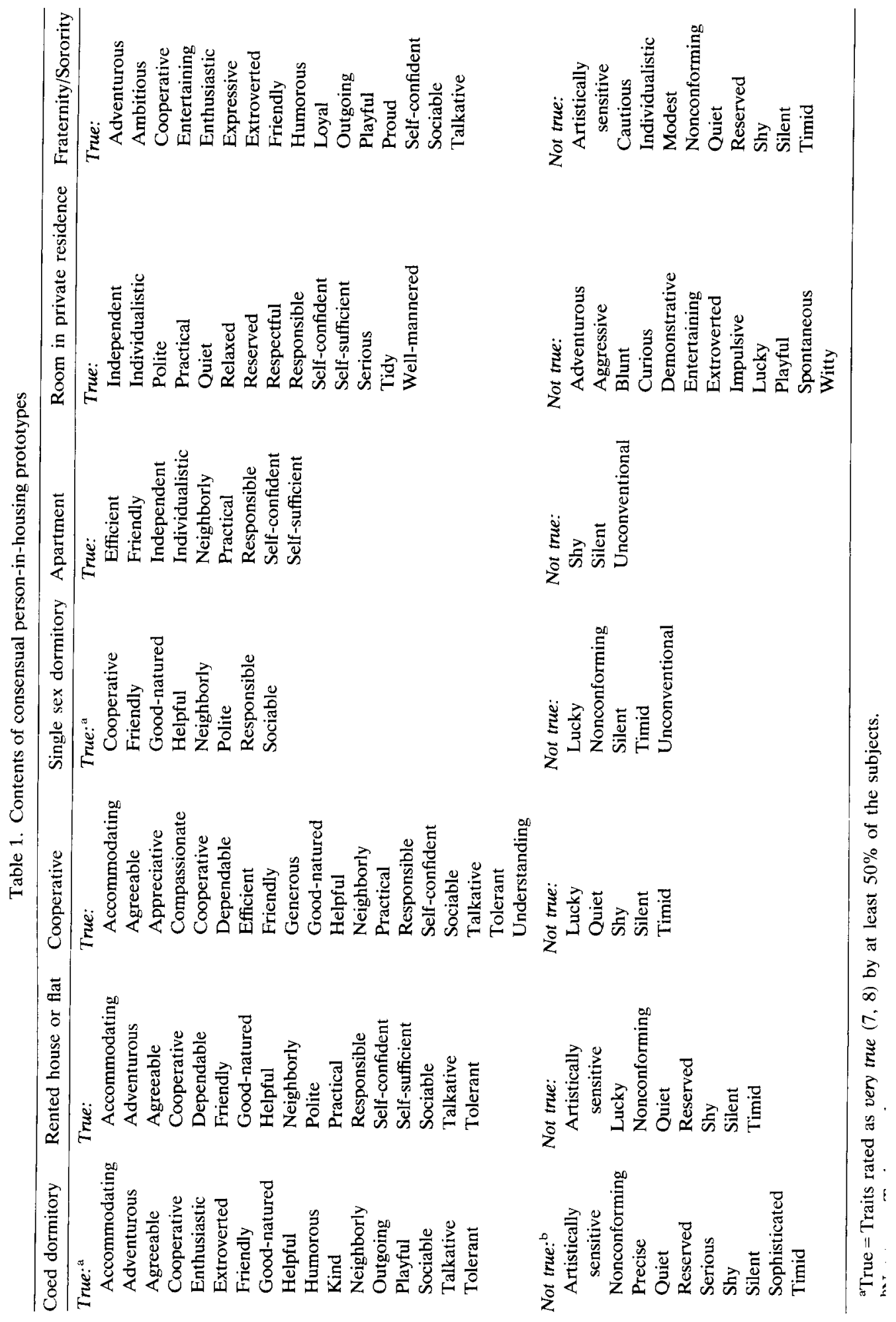


Table 2. Inter-prototype similarity

\begin{tabular}{|c|c|c|c|c|c|c|c|}
\hline \multirow[b]{2}{*}{$\begin{array}{l}\text { Target } \\
\text { prototypes }^{\text {a }}\end{array}$} & \multicolumn{7}{|c|}{ Comparison prototypes } \\
\hline & $\begin{array}{l}\text { Single sex } \\
\text { dormitory }\end{array}$ & Apartment & Coop & $\begin{array}{l}\text { Fraternity/ } \\
\text { Sorority }\end{array}$ & $\begin{array}{l}\text { Room in } \\
\text { private } \\
\text { residence }\end{array}$ & $\begin{array}{l}\text { Coed } \\
\text { dormitory }\end{array}$ & $\begin{array}{l}\text { Rented } \\
\text { house } \\
\text { or flat }\end{array}$ \\
\hline $\begin{array}{l}\text { Single sex } \\
\text { dormitory }\end{array}$ & - & $39 \%$ & $77 \%$ & $46 \%$ & $23 \%$ & $69 \%$ & $85 \%$ \\
\hline Apartment & $42 \%$ & - & $67 \%$ & $33 \%$ & $50 \%$ & $33 \%$ & $67 \%$ \\
\hline $\begin{array}{l}\text { Coop } \\
\text { Fraternity/ }\end{array}$ & $42 \%$ & $29 \%$ & - & $38 \%$ & $17 \%$ & $58 \%$ & $83 \%$ \\
\hline $\begin{array}{l}\text { Sorority } \\
\text { Room in } \\
\text { private }\end{array}$ & $23 \%$ & $15 \%$ & $35 \%$ & - & $4 \%$ & $65 \%$ & $50 \%$ \\
\hline $\begin{array}{l}\text { residence } \\
\text { Coed }\end{array}$ & $12 \%$ & $23 \%$ & $15 \%$ & $4 \%$ & - & $0 \%$ & $23 \%$ \\
\hline $\begin{array}{l}\text { dormitory } \\
\text { Rented } \\
\text { house } \\
\text { or flat }\end{array}$ & $33 \%$ & $15 \%$ & $52 \%$ & $63 \%$ & $0 \%$ & - & $70 \%$ \\
\hline
\end{tabular}

${ }^{\text {aThe }}$ numbers represent the percentage of descriptive traits in the target prototype which are also shared by the comparison prototype.

descriptive of the consensual prototype. These average measures of self-toconsensual-prototype overlap were quite well-reflected in the students' actual housing preferences. For example, $58 \%$ of the students listed apartment as a first or second choice; apartment was also the option with the closest self-toconsensual-prototype match, on average. Similarly, only $14 \%$ of the students listed room in a private residence as a first or second choice; and room was the option with the least self-to-consensual-prototype overlap. Thus, the average self match to the consensual prototypes was reflected in the students' preferences.

\subsection{Individual prototypes and preferences}

So far, we have described students' preferences as a group and have looked at their consensual knowledge of the housing options. We reasoned, however, that predictions about the preferences of particular students would most likely best be made on the basis of each individual's prototypes for the seven housing options. That is, despite the general agreement among the students on the characteristics of the prototypes, individuals certainly differed in some of the specifics of their impressions of the best-suited person for each housing option. So, individual prototypes containing the traits rated as very true $(7,8)$ and very untrue $(1,2)$ by each person for each housing option were constructed (average richness $=22.1$ very true traits and 13.9 very untrue traits). These individual prototypes were used in all further analyses.

The decision rule with which we were most concerned was a self-to-prototype matching rule. We wondered, that is, whether the individual student's preference for any particular housing option would be related to the closeness of her 
self-concept to her prototype for that housing situation. In order to test this hypothesis, we needed a measure of the individual's self-concept which could be compared with his prototype for each housing option. Therefore, we asked students to describe themselves with respect to the same 100 trait adjectives used to construct the person-in-housing prototypes.

Each student's self-concept check list was then matched against each person-insituation prototype in order to find the similarity of the self-to-prototype across the seven options. Specifically, for each option, we subtracted the rating of every adjective on the self-concept check list from the rating of the same adjective in that individual's prototype. We then found the absolute values of the differences and averaged them across the 100 traits to obtain a single 'similarity' score (e.g., the smaller the score, the more similar the self to the prototype) for each self to prototype combination.

Once more we compared the self-prototype comparisons to the housing preference rankings, and once more a telling pattern emerged. A rank order correlation was obtained for each subject for their match of self to prototype similarity and preference of housing option. This rank order correlation was our operational measure of the self-to-prototype matching rule in everyday decision-making. The utility of this rule, and, therefore, the importance of person prototypes in this domain, was reflected in the magnitude of that correlation. In fact, on average, these students' preferences followed the order of their self-to-prototype similarity scores, significantly $\left(r_{\text {corr }}=0.37, p<0.001\right)$. Therefore, across the whole sample of 116 students, the self-to-prototype matching rule was a reasonable, though certainly not perfect predictor of preferences of the seven housing options. As we suspected, housing prototypes were useful in this decision task.

Of course, the decision about university housing concerns factors other than finding a situation in which one would be comfortable as a person. Since, on average, the matching rule rank order correlation was imperfect we suspected that other factors, besides degree of self-to-prototype match, were operating in this decision task (e.g, economic constraints, bad experiences with a particular option, word-of-mouth commentary, and so on). We hypothesized that some students attended more concertedly than did others (perhaps quite automatically and without awareness) to maximizing the fit between their personality attributes, skills and liabilities and those 'required' by the housing situation (i.e., in the housing prototype). The next task, therefore, was to identify this subsample of students, whom we will label the 'maximal rule users'.

\subsection{Maximal use of prototypes}

Who would be inclined to use the rule most? As part of their interview, the students had been asked to list for us the most important factor(s) or piece(s) of information influencing their considerations about housing. Half of the subjects listed economic factors as the most important determinant of their decision (e.g., price of rent and utilities, transportation possibilities). The other half mentioned compatibility with their housemates or neighbors as their main consideration. If an individual is restricted by economic considerations in the housing selection it is 
doubtful that he will consult his store of intuitive social concepts to address the decision task. We reasoned that students whose objectives dealt with socialinterpersonal aspects of housing would draw on their person-in-situation prototypes in making a housing decision. We expected, moreover, that among the sample of students with interpersonal goals differences would exist in the extent to which these prototypes would be implicated in a self-to-prototypes matching strategy. Therefore, tests of the interactions between goals and individual differences in two dimensions of social knowledge were performed.

First, we divided these students $(N=58)$ according to their scores on the Snyder Self-Monitoring Scale [50]. This is a 25 item trait scale which our students had filled out at the end of the interview. Individuals who obtain high scores on this scale (e.g., high self-monitors) are very interested in and attuned to the requirements of different social situations (see [52]). High self-monitors seem to strive quite consistently to 'be the right person in the right situation' and have the skills to do so [51]. Low self-monitors are more conscious of being 'themselves' across situations and less apt to change their behavior to match situational demands. We expected, therefore, that low self-monitors would be more concerned with self-situation matches than the more flexible high self-monitors. This was, in fact, the case. Low self-monitors who had interpersonal goals $(N=18)$ preferred housing whose prototype they matched (average $r_{\text {corr }}=0.54$ ) far more often than did the high $(N=17)$ self-monitors with interpersonal goals (average $r_{\text {corr }}=0.20, p=0.03$ ). A similar difference between high and low selfmonitors, in use of the self-to-prototype matching rule, was not observed in the subsample of students with practical goals. In our second analysis of the utility of the self-to-prototype matching rule, we looked at the students with interpersonal goals who also had rated many traits as not at all true of themselves (a prototype of self knowledge we call 'high distinctiveness'). We hypothesized that if an individual has interpersonal goals and yet knows that many traits are very untrue of himself, that individual will be particularly anxious to find a 'good' housing match. The individual who 'knows' that many traits are clearly untrue of himself may also "know" that he will have to work harder to fulfill those interpersonal goals, i.e., to find a 'good' self-housing fit. To test this hypothesis, we divided students into subgroups: students who had rated many traits as not at all true of themselves, relative to the total sample of students (High Distinctiveness); and students with interpersonal goals who had rated relatively few traits as not at all true of themselves (Low Distinctiveness). Again, our suspicions proved correct. Students with low distinctiveness scores and interpersonal goals $(N=28)$ used a self-to-prototype matching rule less often (average $r_{\text {corr }}=0.28$ ) than did the students with the high distinctiveness $(N=30)$ scores (average $r_{\text {corr }}=0.52$ ). This difference in the degree to which these students' preferences matched their selfto-prototype similarity scores was statistically significant $(p=0.05)$. Again, this difference did not obtain for students with practical goals.

The foregoing analyses suggest that not only can person prototypes be useful to everyone in such a decision task, but they can be especially useful to "some of the people, some of the time" [3]. It seems that in analyzing the utility of prototypes in naturalistic decision making, it is crucial to pay attention to the goals of the 
individuals who are engaged in the decision task. The importance of individuals' goals in shaping social cognition has only recently begun to receive the attention which it deserves $[21,23]$.

In the present study, we were concerned with individuals' social knowledge and skills in a naturalistic decision task. The domain of interest, university housing choices, was actually quite a complex one: We focused upon one set of knowledge - prototypes of suitable person - housing fit - and one decision ruleself-to-prototype matching. The comparisons between average self-prototype match across the sample and overall preferences made it clear that person prototypes could be important in this task. On an individual level, our actual test of use of the prototype matching rule was a quite rigorous one. In order to obtain a substantial rank order correlation between self-to-prototype similarity and housing preferences, a student had to demonstrate articulate knowledge of the self in relation to all seven prototypes and then use that knowledge to rank his preferences by goodness-of-self-fit in all seven situations. The rank order correlation, our test of the rule, would have been reduced considerably if an individual eliminated even a few of the options out-of-hand, on the basis of factors other than self-prototype match-for example, word-of-mouth reputation, financial constraints, and so forth. Therefore, in acknowledging the difficulty of using a self-to-prototype matching rule by these standards, we were encouraged by the significant patterns (of rank order correlations) that did emerge from these data.

\section{Conclusions}

Why do we categorize objects and persons at all, if we can only partition them into fuzzy sets nested within loosely structured taxonomies? Doesn't categorization do more harm than good, create more 'mess' than order, especially in the person domain? The present review has developed our contention that, contrary to intuition, 'fuzziness' does not imply 'messiness' or 'uselessness'. Even in the person domain, an area in which experts have struggled to construct formal taxonomies, the orderliness and utility of fuzzy natural categories can be documented.

We introduced our argument for the theoretical utility of natural categories with the notion of a category prototype. That is, though they lack defining features and clear-cut boundaries, natural categories can be unified and organized around the representative or prototypic category exemplars. Some category exemplars better epitomize the essential meaning of the concept than others; these prototypes can serve as reference points in the identification of 'new' category members. A continuum of prototypicality of membership, as well as consensual rules for judging goodness-of-fit in the category, have been documented for natural categories ranging from birds to extroverts to parties. Thinking about prototypes brings order to the structure of fuzzy categories.

If the structure of natural categories can be simplified or clarified by focusing on prototypic exemplars, so, too, can the mental representations for the categories. The central meaning, as well as the heterogeneity of membership in a category 
can be nicely captured in a prototype representation. The prototype may take the form of a collection of 'classic' category exemplars or it may be a summary or abstraction of the characteristic features of category members. Regardless of the exact form, and both representations may exist, the prototype helps summarize the central tendency and the variability in the features of category members. In so doing, the prototype reduces an enormous quantity of information into a rather neat capsule summary; that representation can then help the perceiver to identify "new' category members - to answer the question, "what is that?" And, in the domain of persons, the prototype also provides information about the likely behavior of this 'other person', so as to facilitate quick responses and ease social interaction. How else would you 'know' in advance the possible outcomes of an encounter with a hippy?

Finally, if the structure is orderly and the mental representation is rich yet economical, then why not use natural categories in everyday life? Well, we do, or so it seems. Natural categories are useful not only in making identifications and predictions about potential category members, but also in making personal decisions about products to buy, people with whom to affiliate, and houses in which to live. By using the information in prototypes, individuals can test the match of a person, place or object to their own tastes quite easily. This was supported by the case analysis of students' preferences for university housing. In that analysis, the match between students' own characteristics and those of the person whom they imagined best-fit each particular housing option (a person-inhousing prototype) was significantly related to preference in housing. Naturalistic decision-making can be eased through the use of a self-to-prototype matching rule, in order to differentiate between 'good' and 'poor' options. People even share such prototypes for car owners - are you the typical 'Toyota type', 'BMW type' or 'Ford type'? It is quite useful in the business of living to know your person prototypes.

\section{Acknowledgment}

Preparation of this manuscript was supported by Grant \#BNS-8022253 from the National Science Foundation. We wish to thank Sara Freeland for her help in preparation of this manuscript.

\section{References}

[1] R.P. Abelson, Psychological status of the script concept, American Psychologist 36 (1981) 715-729.

[2] S.L. Armstrong, H. Gleitman and L.R. Gleitman, What concepts might not be, Cognition, in press.

[3] D.J. Bem and A. Allen, On predicting some of the people some of the time: The search for cross-situational consistencies in behavior, Psychological Review 81 (1974) 506-520.

[4] D.J. Bem and D. Funder, Predicting more of the people more of the time: Assessing the personality of situations, Psychological Review 85 (1978) 485-502.

[5] S.L. Bem, The measurement of psychological androgyny, Journal of Consulting and Clinical Psychology 42 (1974) 115-162. 
[6] M.B. Brewer, V. Dull and L. Lui, Perceptions of the elderly: Stereotypes as prototypes, Journal of Personality and Social Psychology 41 (1981) 656-670.

[7] J.S. Bruner, J. Goodnow and G. Austin, A Study of Thinking (Wiley, New York, 1956).

[8] D.B. Buss and K.H. Craik, The act frequency approach to personality, Psychological Review 90 (1983) 105-126.

[9] N. Cantor, Prototypicality and personality judgments, Unpublished Doctoral Dissertation, Stanford University (1978).

[10] N. Cantor, Perceptions of situations: Situation prototypes and person-situation prototypes, in: D. Magnusson, Ed., The Situation: An Interactional Perspective (Lawrence Erlbaum, Hillsdale, NJ, 1980).

[11] N. Cantor and N. Genero, Psychiatric diagnosis and natural categorization: A close analogy, in: T. Millon and G. Klerman, Eds., Contemporary Issues in Psychopathology (Guildford Press, New York, in press).

[12] N. Cantor and J.F. Kihlstrom, Social intelligence: The cognitive basis of personality, Technical Report No. 60, Cognitive Science Center, University of Michigan (1983).

[13] N. Cantor, D. Mackie and C. Lord, Choosing partners and activities: The social perceiver decides to mix it up, Social Cognition 2 (1984).

[14] N. Cantor and W. Mischel, Traits as prototypes: Effects on recognition memory, Journal of Personality and Social Psychology 35 (1977) 38-48.

[15] N. Cantor and W. Mischel, Prototypes in person perception, in: L. Berkowitz, Ed., Advances in Experimental Social Psychology, Vol. 12 (Academic Press, New York, 1979).

[16] N. Cantor, W. Mischel and J. Schwartz, A prototype analysis of psychological situations, Cognitive Psychology 14 (1982) 45-77.

[17] N. Cantor, E. Smith, R. de S. French and J. Mezzich, Psychiatric diagnosis as prototype categorization, Journal of Abnormal Psychology 89 (1980) 181-193.

[18] L.J. Chapman and J.P. Chapman, Illusory correlations as an obstacle to the use of valid psycho-diagnosis signs, Journal of Abnormal Psychology 74 (1960) 271-280.

[19] H.H. Clark and E.V. Clark, Psychology and Language (Harcourt Brace Jovanovich, New York, 1977).

[20] C.E. Cohen, Person categories and social perception: Testing some boundaries of the processing effects of prior knowledge, Journal of Personality and Social Psychology 40 (1981) 441-452.

[21] C.E. Cohen, Goals and schemata in person perception: Making sense from the stream of behavior, in: N. Cantor and J.F. Kihlstrom, Eds., Personality, Cognition and Social Interaction (Lawrence Erlbaum, Hillsdale, NJ, 1981).

[22] D.L. Hamilton, A cognitive-attributional analysis of stereotyping, in: L. Berkowitz, Ed., Advances in Experimental Social Psychology, Vol. 12 (Academic Press, New York, 1979).

[23] E.T. Higgins and G. King, Accessibility of social constructs: Information-processing consequences of individual and contextual variation, in: N. Cantor and J.F. Kihlstrom, Eds., Personality, Cognition and Social Interaction (Lawrence Erlbaum, Hillsdale, NJ, 1981).

[24] C. Hoffman, W. Mischel and K. Mazze, The role of purpose in the organization of information about behavior: Trait-based versus goal-based categories in person cognition, Journal of Personality and Social Psychology 40 (1981) 211-225.

[25] K.S. Holyoak and P.C. Gordon, Social reference points, Journal of Personality and Social Psychology 44 (1983) 881-887.

[26] E.E. Jones, D.G. Kanouse, H.H. Kelley, R.E. Nisbett, S. Valins and B. Weiner, Eds., Attribution: Perceiving the Causes of Behavior (General Learning Press, Morristown, NJ, 1972).

[27] D. Kahneman and A. Tversky, On the psychology of prediction, Psychological Review 80 (1973) $237-251$.

[28] J.F. Kihlstrom and C. Chew, Adjective Checklist, Unpublished Data, University of Michigan (1981).

[29] D.R. Kinder, M.D. Peters, R.P. Abelson and S.T. Fiske, Presidential prototypes, Political Behavior 2 (1980) 315-337.

[30] N.A. Kuiper and T.B. Rogers, The encoding of personal information: Self-other differences, Journal of Personality and Social Psychology 37 (1979) 449-514. 
[31] H. Markus, Self-schemata and processing information about the self, Journal of Personality and Social Psychology 35 (1977) 63-78.

[32] H. Markus and J. Smith, The influence of self-schemata on the perception of others, in: N. Cantor and J.F. Kihlstrom, Eds., Personality, Cognition and Social Interaction (Lawrence Erlbaum, Hillsdale, NJ, 1981).

[33] M. McCloskey and S. Glucksberg, Natural categories: Well defined or fuzzy sets, Memory and Cognition 6 (1978) 462-472.

[34] C. Mervis, J. Catlin and E. Rosch, Development of the structure of color names, Developmental Psychology 11 (1975) 54-60.

[35] J. Money and A.A. Ehrhardt, Man and Woman; Boy and Girl (Johns Hopkins Press, Baltimore, 1972).

[36] P.M. Niedenthal, N. Cantor and J.F. Kihlstrom, Prototype-matching: A strategy for social decision-making, Unpublished manuscript, University of Michigan (1983).

[37] V. Neisser, The concepts of intelligence, Intelligence 3 (1979) 217-227.

[38] W.T. Norman, Toward an adequate taxonomy of personality attributes: Replicated factor structure in peer nomination personality ratings, Journal of Abnormal and Social Psychology 66 (1963) 574-583.

[39] M. Posner and S. Keele, On the genesis of abstract ideas, Journal of Experimental Psychology 77 (1968) 353-363.

[40] G.A. Quattrone and E.E. Jones, The perception of variability within in-groups and out-groups: Implications for the law of small numbers, Journal of Personality and Social Psychology 38(1) (1980) 141-152.

[41] S.K. Reed, Pattern recognition and categorization, Cognitive Psychology 3 (1972) 382-407.

[42] L.J. Rips, E.J. Shobin and E.E. Smith, Semantic distance and the verification of semantic relations, Journal of Verbal Learning and Verbal Behavior 12 (1973) 1-20.

[43] E. Rosch, Principles of categorization, in: E. Rosch and B.B. Lloyd, Eds., Cognition and Categorization (Lawrence Erlbaum, Hillsdale, NJ, 1978).

[44] E. Rosch and B.B. Lloyd, Editors, Cognition and Categorization (Lawrence Erlbaum, Hillsdale, NI, 1978).

[45] E. Rosch and C. Mervis, Family resemblance: Studies in the internal structure of categories, Cognitive Psychology 7 (1975) 513-605.

[46] E. Rosch, C. Mervis, W. Grey, D. Johnson and P. Boyes-Braem, Basic objects in natural categories, Cognitive Psychology 8 (1976) 382-439.

[47] L. Ross, The intuitive psychologist and his shortcomings: Distortions in the attribution process, in: L. Berkowitz, Ed., Advances in Experimental Social Psychology, Vol. 10 (Academic Press, New York, 1977).

[48] E.E. Smith and D.L. Medin, Categories and Concepts (Harvard University Press, Cambridge, Mass., 1981).

[49] E.E. Smith, E.J. Shobin and L.J. Rips, Structure and process in semantic memory: A featural model for semantic decisions, Psychological Review 81 (1974) 214-241.

[50] M. Snyder, Self-monitoring of expressive behavior, Journal of Personality and Social Psychology 30 (1974) 526-537.

[51] M. Snyder, Self-monitoring processes, in: L. Berkowitz, Ed., Advances in Experimental Social Psychology, Vol. 12 (Academic Press, New York, 1979).

[52] M. Snyder, On the influence of individuals on situations, in: N. Cantor and J.F. Kihlstrom, Eds., Personality, Cognition and Social Interaction (Lawrence Erlbaum, Hillsdale, NJ, 1981).

[53] A. Tversky, Features of similarity, Psychological Review 84 (1977) 327-352.

[54] J.S. Wiggins, A psychological taxonomy of trait-descriptive terms: The interpersonal domain, Journal of Personality and Social Psychology 37(3) (1979) 395-413.

[55] L. Wittgenstein, Philosophical Investigations (Macmillan, New York, 1953). 